\title{
BIOLOGÍA REPRODUCTIVA DE «BOQUICHICO», Prochilodus nigricans, EN LA AMAZONÍA PERUANA
}

\author{
Víctor Montreuil", Áurea García*, Ronald Rodríguez
}

\section{RESUMEN}

La época de reproducción de «boquichico», Prochilodus nigricans, está comprendida entre los meses de diciembre y marzo. Este periodo coincide con el inicio del aumento de aguas del río Amazonas y no guarda mayor relación con el incremento de las precipitaciones locales. La especie en cuestión alcanza su talla media de madurez a una longitud a la horquilla de $24,3 \mathrm{~cm}$, en el caso de las hembras, y de $23,4 \mathrm{~cm}$ en el caso de los machos.

Palabras clave: Madurez sexual, «boquichico», Prochilodus nigricans, Amazonía Peruana.

\begin{abstract}
Reproduction of «boquichico» Prochilodus nigricans, at the Peruvian Amazon takes place from December to March, coinciding with the period of water rising of the Amazon River, not showing a clear relationship with the increment of local raining. This species reaches its average size of maturity at a fork length of $24,3 \mathrm{~cm}$., in females, and 23,4 cm., in males.
\end{abstract}

Key words: $\quad$ Sexual maturity, «boquichico», Prochilodus nigricans, Peruvian Amazon.

\section{INTRODUCCIÓN}

En la Amazonía Peruana, la pesquería de consumo humano se sustenta, fundamentalmente, en especies de vida corta, rápido crecimiento y alta fecundidad. Dentro de ellas el «boquichico» es la de mayor importancia, pues representa el $44,7 \%$ de la captura comercial desembarcada en Iquitos.

* Instituto de Investigaciones de la Amazonía Peruana, Programa de Ecosistemas Acuáticos, Centro Regional de Investigaciones Loreto, Apartado Postal 784, Iquitos - Perú. 
Por razones no del todo explicadas hasta el momento, esta especie ha comenzado a mostrar algunos signos preocupantes, como la reducción del tamaño promedio de captura y el creciente alejamiento de las zonas de pesca. Estos hechos se suman a la falta de estrategias de manejo de las poblaciones de Prochilodus nigricans, que aseguren una producción permanente a través del tiempo.

En este sentido, con el objetivo de proporcionar información relevante para la regulación de la captura de esta especie, hemos iniciado el estudio de su ciclo de maduración, de su época de reproducción y de la influencia de los factores climáticos estacionales sobre estos parámetros biológicos.

\section{MATERIALES Y MÉTODOS}

El material estudiado fue obtenido a través de un muestreo de los desembarques de la flota pesquera comercial de Iquitos y Nauta durante el periodo comprendido entre enero y diciembre de 1993. Se analizó un total de 650 ejemplares de «boquichico», Prochilodus nigricans, provenientes del bajo Amazonas peruano (Orán, Apayacu, Porvenir, Shishita) y del río Marañón (San Pedro Tipishca, 23 de Junio, Cocha Shiriyacu y Paranapura).

Se registró la longitud a la horquilla de cada ejemplar en centímetros utilizando un ictiómetro de $50,0 \mathrm{~cm}$ de longitud, graduado a $0,2 \mathrm{~mm}$. El peso total de cada individuo se registró, en gramos, con una balanza Ohaus de $2610 \mathrm{~g}$ con $0,1 \mathrm{~g}$ de sensibilidad.

Realizando un corte ventral, desde la altura del opérculo hasta el orificio anal, se determinó el sexo por exposición de la cavidad visceral. Las gónadas fueron analizadas y pesadas en fresco en el laboratorio. El peso de la gónada se determinó usando una balanza Ohaus con aproximación de $0,1 \mathrm{~g}$.

Se elaboró una escala de madurez sexual para ambos sexos considerando las características externas de las gónadas, como longitud y ancho $(\mathrm{cm})$, peso $(\mathrm{g})$, irrigación sanguínea, textura, color y presencia o ausencia de productos sexuales. Para esto, se consideraron las escalas propuestas por Nair de Buckman (Laevastu, 1971); Ráez (1976), citado por Araujo (1985), y Tello et al. (1992).

El índice gonadosomático se calculó usando la ecuación descrita en Tresierra y Culquichicón (1993). 
$\mathrm{IGS}=\frac{\mathrm{Pg}}{\mathrm{Pp}} \times 100$

Donde:

IGS = Índice gonadosomático

$\mathrm{Pg}=$ Peso de la gónada

$\mathrm{Pp}=$ Peso del pez

La talla media de madurez (Tmm) se encontró agrupando los ejemplares maduros de talla de $2 \mathrm{~cm}$ por sexo y por clase. Luego de ello, se determinó la frecuencia acumulada porcentual de cada clase.

La época de desove fue identificada mediante el análisis de las variaciones del índice gonadosomático a través del tiempo.

La relación entre la época de reproducción y los factores climáticos (precipitaciones y nivel del río Amazonas) fue analizada para determinar la influencia de dichos factores en el comportamiento reproductivo de la especie en estudio.

Complementariamente, se calculó la talla media de captura con el fin de realizar comparaciones con la Tmm.

\section{RESULTADOS}

De acuerdo con las características macroscópicas de las gónadas, se estableció la escala de maduración sexual para Prochilodus nigricans (Tabla 1).

\section{Talla media de maduración}

La longitud en que el 50\% de hembras y machos de «boquichico» alcanza la talla media de maduración se presenta en las figuras 1 y 2 . Puede observarse que las hembras maduran a los 24,3 cm, mientras que los machos lo hacen a los $23,4 \mathrm{~cm}$.

\section{Época de maduración y desove}

El periodo reproductivo se inicia en diciembre y se prolonga hasta marzo. En diciembre, el IGS alcanza un valor de 7,9\%, lo que indica el inicio de la maduración sexual. 
Tabla 1. Escala de los estadios de madurez sexual.

\begin{tabular}{|c|c|}
\hline Estadio de madurez sexual & Características externas (gónadas) \\
\hline $\begin{array}{l}\text { I } \\
\text { Virgen }\end{array}$ & $\begin{array}{l}\text { Órganos sexuales (testículos y ovarios) bastante } \\
\text { pequeños, cerca y debajo de la columna vertebral, } \\
\text { transparentes. Óvulos no visibles. }\end{array}$ \\
\hline $\begin{array}{l}\text { II } \\
\text { Maduración inicial }\end{array}$ & $\begin{array}{l}\text { Testículos y ovarios de color rojo pálido, más de- } \\
\text { sarrollados que en el estadio anterior. Óvulos no } \\
\text { visibles. }\end{array}$ \\
\hline $\begin{array}{l}\text { III } \\
\text { Maduración intermedia }\end{array}$ & $\begin{array}{l}\text { Testículos opacos, rojizos. Ovarios de color ana- } \\
\text { ranjado rojizo con irrigación sanguínea poco per- } \\
\text { ceptible. Los óvulos se observan como pequeños } \\
\text { puntos blanquecinos. }\end{array}$ \\
\hline $\begin{array}{l}\text { IV } \\
\text { Maduración avanzada }\end{array}$ & $\begin{array}{l}\text { Testículos blanco-rojizos. No aparecen gotas de } \\
\text { semen que ejerzan presión. Los ovarios son de } \\
\text { color amarillo grisáceo con óvulos visibles. Los } \\
\text { testículos y ovarios ocupan más de la mitad de la } \\
\text { cavidad ventral. }\end{array}$ \\
\hline $\begin{array}{l}\mathrm{V} \\
\text { Maduro }\end{array}$ & $\begin{array}{l}\text { Los órganos sexuales ocupan casi toda la cavidad } \\
\text { ventral. Testículos totalmente blancos. Las gotas } \\
\text { de semen caen cuando se presiona fuertemente. } \\
\text { Ovarios de color verde cobre con irrigación san- } \\
\text { guínea bien acentuada y óvulos grandes, comple- } \\
\text { tamente redondos. }\end{array}$ \\
\hline $\begin{array}{l}\text { VI } \\
\text { En desove }\end{array}$ & $\begin{array}{l}\text { Los órganos sexuales llenan la cavidad ventral. } \\
\text { Membrana ovárica débil. Los óvulos salen al ex- } \\
\text { terior ante una ligera presión del abdomen. El lí- } \\
\text { quido espermático se libera con suma facilidad. }\end{array}$ \\
\hline $\begin{array}{l}\text { VII } \\
\text { Desovado }\end{array}$ & $\begin{array}{l}\text { Órganos sexuales de color blanco rojizo y rojo } \\
\text { vinoso, flácidos, semejantes a sacos vacíos. }\end{array}$ \\
\hline
\end{tabular}


En enero, el IGS evoluciona hasta un valor máximo de 15,6\%. En este momento, se produce una caída paulatina, que marca el inicio del periodo de desove. Este último concluye en los primeros días de abril cuando se registra un IGS de $0,5 \%$, que se mantiene estacionario hasta noviembre (Figura 3).

Figura 1. Talla media de maduración de «boquichico». Hembras.

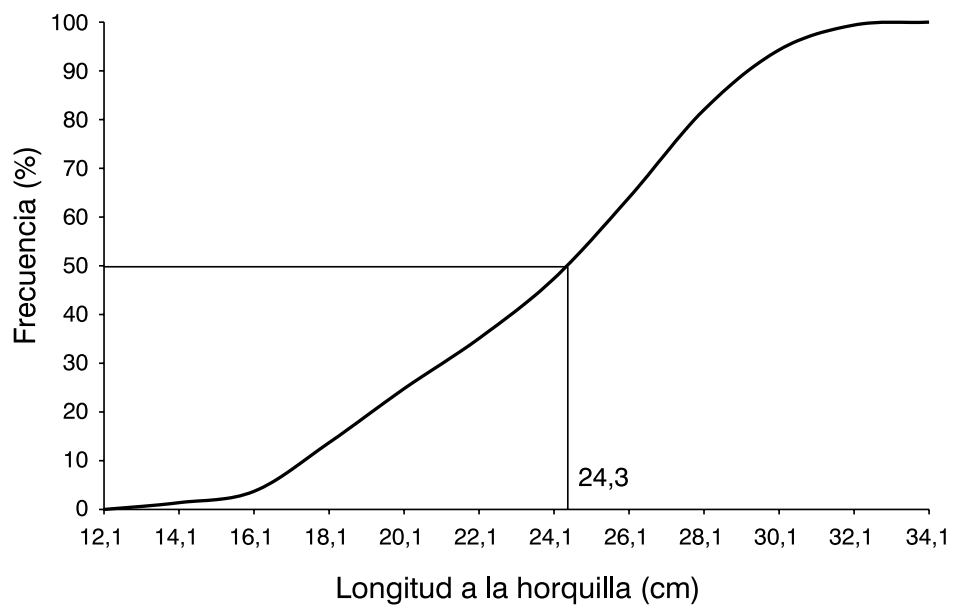

Figura 2. Talla media de maduración de «boquichico». Machos.

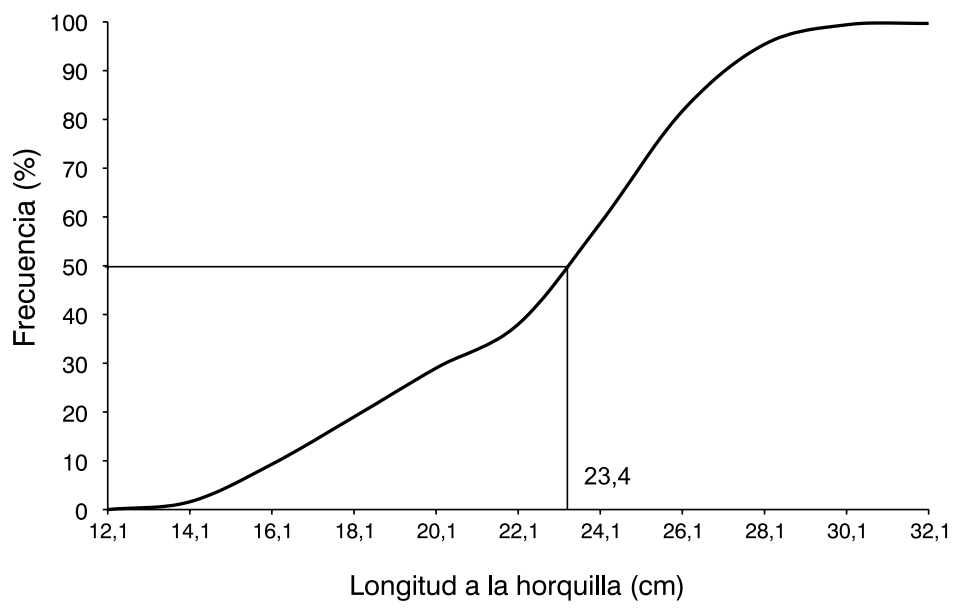


Si se relaciona la época de reproducción con los factores climáticos (nivel del río y precipitaciones), se puede apreciar que el desove de la especie en estudio está vinculado al aumento del nivel del río antes de que este alcance su máximo nivel. Ahora bien, este hecho no muestra una aparente relación con las precipitaciones locales (Figuras 4 y 5).

Figura 3. Época de maduración y desove (IGS) de «boquichico».

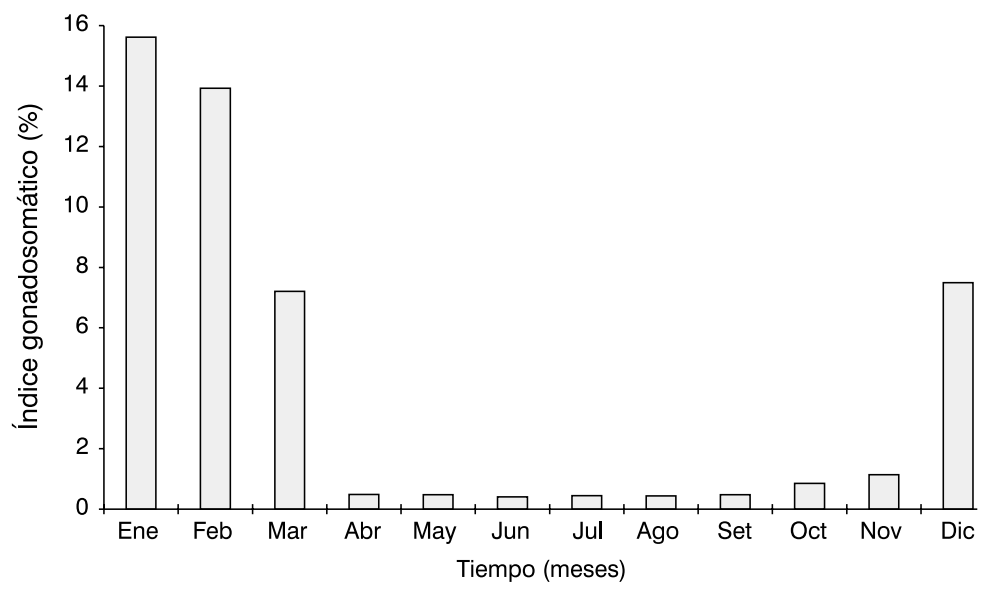

Figura 4. Época de desove de «boquichico» en relación con las variaciones del nivel del río.

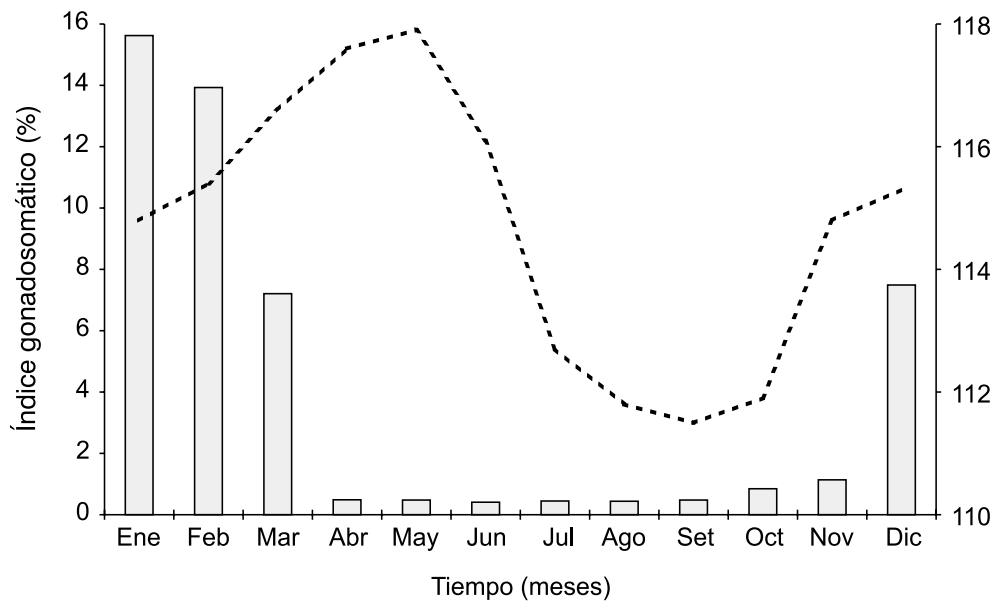


Figura 5. Época de desove de «boquichico» en relación con las precipitaciones.

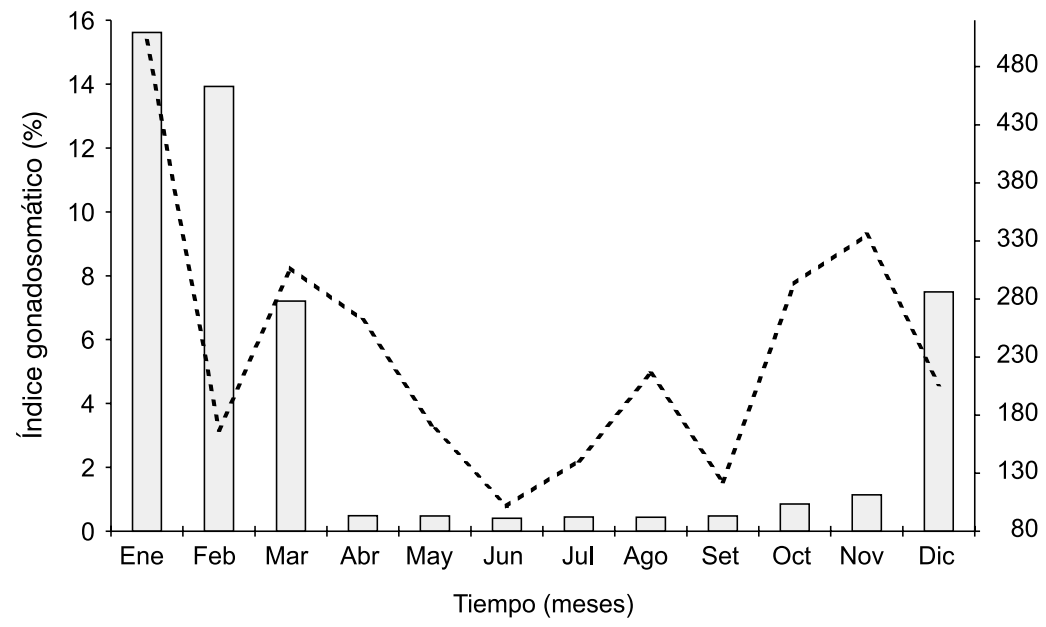

\section{DISCUSIÓN}

El análisis de la información disponible para este reporte nos permite concluir que la longitud media de maduración sexual de Prochilodus nigricans es de $24,3 \mathrm{~cm}$ de longitud a la horquilla en las hembras y de $23,4 \mathrm{~cm}$ en los machos. Estos resultados discrepan de aquellos encontrados por Lauzanne et al. (1990) para la misma especie en el río Mamoré, Bolivia. Según este autor, las hembras maduran a una talla más pequeña $(26,0 \mathrm{~cm})$ que los machos $(27,0 \mathrm{~cm})$. Aunque existen diferencias en el tipo de longitud utilizada (longitud total) por Lauzanne, por el momento, no podemos hipotetizar razones que expliquen las variaciones relativas al sexo.

Welcomme (1979), citado por Galvis et al. (1989) y Novoa y Ramos (1982) indican que la reproducción de muchos peces en los ríos tropicales es altamente estacional y que, en los ríos que presentan variaciones en su nivel a través del año, ocasionadas por regímenes hidrológicos variables, existe una sincronización entre los procesos reproductivos de la mayoría de peces y el incremento en el nivel de las aguas.

En Prochilodus nigricans, se ha verificado la presencia de una época de reproducción que se prolonga desde diciembre hasta marzo con un pico máximo en enero. Este lapso coincide con el periodo de aguas altas del río Amazonas, comportamiento que también fue observado, para otras especies, por Guevara (1974), Basile-Martins (1978), Caramaschi (1979) y Tello et al. (1992). 
La estrategia de reproducción durante las aguas altas favorece mucho a las larvas y alevinos que encuentran, en coincidencia a lo expuesto por Lauzanne et al. (1990), diversos ambientes para su protección natural en las zonas inundadas, así como también adecuadas condiciones para su alimentación y desarrollo.

Aunque aún son evidentes hechos como la reducción de la talla promedio de comercialización y la escasez de ejemplares de esta especie en zonas de pesca tradicionales cercanas a Iquitos, se ha encontrado, a través de este estudio, que la talla promedio de captura $(24,7 \mathrm{~cm}$ longitud a la horquilla) es superior a la talla de primera maduración, tanto de los machos como de las hembras. Desafortunadamente, no existe información previa que nos permita confrontar, en una escala temporal, las diferencias entre tallas promedios de captura y tallas medias de maduración.

\section{BIBLIOGRAFÍA}

ARAUJO, R. 1985. Estudio del procesamiento de «boquichico», Prochilodus nigricans, ahumado en caliente. Lima (Perú): Universidad Nacional Agraria La Molina. Facultad de Pesquería. Tesis de Ingeniero Pesquero. 102 pp.

BASILE-MARTINS, M.A. 1978. Comportamiento e alimentaçao de Pimelodus maculatus Lacéde 1803 (Osteichthyes, Siluriformes, Pimelodidae). Tese de Doutor. Sao Paulo: Departamento de Zoología do Instituto de Biociencias da Universidad de Sao Paulo.

CARAMASCHI, E. 1979. Reproduçao e alimentaçao de Hoplias malabaricus (Block 1794), na represa do rio Pardo (Botucatu, SP) (Osteichthyes, Cycriniformes, Erythrinidae). Sao Carlos, Sao Paulo: Universidad de Sao Carlos. Departamento Ciencias Biológicas. Tese de Mestrado.

GALVIS, G.; MOJICA, J.; RODRÍGUEZ, F. 1989. Estudio ecológico de una laguna de desborde del río Metica - Orinoquia Colombiana. Centro Editorial Universidad Nacional de Colombia, Fondo Fen Colombia.

GARCÍA, A.; RODRÍGUEZ, R. 1995. Madurez sexual de «boquichico» Prochilodus nigricans. Iquitos (Perú): Universidad Nacional de la Amazonía Peruana. Tesis de Biólogo. 
GUEVARA, J. 1974. Estudio sobre el ciclo biológico, ecología, etología y crianza experimental de sábalos (Pisces, Characidae) en la Amazonía Peruana. Lima (Perú): Universidad Nacional Mayor de San Marcos. Tesis de Bachiller en Biología.

LAEVASTU, T. 1971. Manual de métodos de biología pesquera. Zaragoza (España): Editorial Acribia. 243 pp.

LAUZANNE, L.; LOUBENS, G.; LE-GUENNEC, B. 1990. Pesca y biología pesquera en el Mamore medio (Región de Trinidad Bolivia). En: Interciencia. Vol. 15. $\mathrm{N}^{\mathrm{o}} 6.452-460 \mathrm{pp}$.

NOVOA, D.; RAMOS, S. 1982. (Comp.). Los recursos pesqueros del río Orinoco y su explotación. Caracas (Venezuela): Editorial Arte. 358 pp.

TELLO, S.; MONTREUIL, V.; MACO, J.; ISMIÑO, R.; SÁNCHEZ, H. 1992. Bioecología de peces de importancia económica de la parte inferior de los ríos Ucayali y Marañón. En: Folia Amazónica. Vol. 2. 75-93 pp.

TRESIERRA, A.; CULQUICHICÓN, Z. 1993. Biología pesquera. Trujillo (Perú): Editorial Libertad. 